\title{
THE FASCICULUS CEREBRO-SPINALIS IN THE ALBINO RAT
}

\author{
S. WALTER RANSON
}

The Anatomical Laboratory of the Northweslern University Medical School

TEN FIGURES

It is well known that the fasciculus cerebro-spinalis, more commonly called the cortico-spinal or pyramidal tract, does not occupy the same position in the spinal cord in all orders of mammals. But, according to the animal which is being studied, it may be found in any one or two of the three funiculi of the cord. From its constant position in the ventro-medial portion of the medulla it passes in the rat to the opposite posterior funiculus of the spinal cord; while in the mole it runs without decussation into the anterior funiculus of the same side. In the cat it decussates into the opposite lateral funiculus, while in man a part of the fibers go over into the opposite lateral funiculus and a smaller part run without decussation into the homolateral anterior funiculus.

There have been published a large number of articles dealing with such variations in the position of the pyramidal tract, and with the corresponding variations in the pyramidal decussation; but very little attention has been paid to the character of the fibers of which this fasciculus is composed. A study of sections of the spinal cord of the rat, guinea-pig, rabbit and cat prepared by the puridine silver technique has brought to light great differences in the pyramidal fibers in these different animals. The variations in the size of the axons and in the degree to which the myelin sheaths are developed are no less striking, and probably more significant, than the variations in the position which the tract as a whole assumes. It is with the characteristics of these fibers in the white rat that this paper is primarily concerned and we 
hope to follow it with a comparative study of the pyramidal fibers in several different orders of mammals. For this reason no attempt will be made at this time to give a comprehensive review of the literature.

Stieda ('69) noticed that the pyramidal tract in the mouse occupied the posterior funiculus. Spitzka ('86) showed that this position was characteristic for the rat and the guinea-pig. These observations were confirmed on the rat by Von Lenhossék ('89) and Bechterew ('90), using the embryological method of Fleichsig. Further confirmation was obtained through the application of the Marchi stain to the degenerating tract in the rat by Goldstein ('04), Van der Vlort ('06) and Miss King ('10).

\section{TECHNIQUE}

In this investigation the pyridine-silver (modified Cajal) technique (Ranson '12) was used as the principal method and the results were controlled by the use of the Weigert and the PalWeigert methods. For the Weigert methods some of the cords were fixed in Müller's fluid and others after fixation in 10 per cent formalin were mordanted either in Müller's fluid or in the following solutions:

Primary mordant

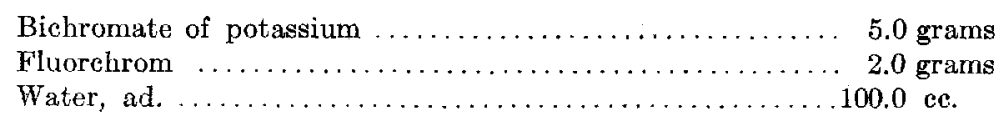

Secondary mordant

Acetate of copper...................... 5.0 grams

Acetic acid ( 35 per cent) $\ldots \ldots \ldots \ldots \ldots \ldots \ldots \ldots \ldots \ldots . \ldots \ldots$ grams

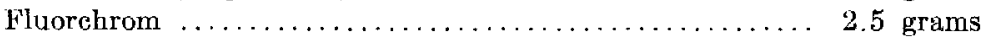

Water, ad............................. $100.0 \mathrm{ce}$.

The usual staining and differentiating solutions were employed and paraffin as well as celloidin sections were utilized. An effort was made to use as many different combinations as possible, and in this way to exclude the possibility that the characteristic staining of the pyramidal tracts was due to theparticular modification of the method employed. 
The pyramidal fasciculi in the white rat take a very light stain with the Weigert methods, appearing under low magnification as grayish blue areas clearly marked off from the remainder of the white substance which stains a deep blue. On the other hand, the pyridine-silver technique causes these tracts to stand out from the rest of the cord because of the dark brown color which they assume. Since the remainder of the white substance stains a very light brown, the contrast is striking and could be equalled only by the most fortunate Marchi preparations. This contrast is equally evident in the decussation and after the pyramidal tracts have assumed their position on the ventral surface of the medulla.

Since nowhere in the literature are to be found altogether satisfactory figures and descriptions of the position and shape of the pyramidal tracts at different levels of the rat's medulla and spinal cord, it seems desirable again to go over these purely topographical features before taking up the finer structure.

\section{TOPOGRAPHY}

The changes in shape, size, and position of the tract at various levels can best be described in connection with figures 1 to 7 . Figure 1 was drawn from a section through the upper end of the decussation of the pyramids. At and above this level in the medulla the pyramids are situated on either side of the anterior median sulcus, but do not project ventrally as they do in thehuman brain. Fibers can be seen detaching themselves from the pyramids and running backwards to decussate as small bundles, or as individual fibers. On reaching the gray substance they spread our rather diffusely in the form of small branching bundles, many of whose fibers end within the medulla at the level of their decussation.

Where the decussation is at its height (fig.2) the crossing bundles are of large size. They run backward at some distance from the central canal, and are gathered together on the dorsal surface of the gray substance into two large fasciculi. These are at first some distance apart, but approach each other in the lower part of the medulla. Figure 3 represents the lowest level of the 
With the exception of figures 5 and 9 , which are from Pal-Weigert preparations, the drawings were made from pyridine-silver preparations. All drawings were made with a Leitz microscope.
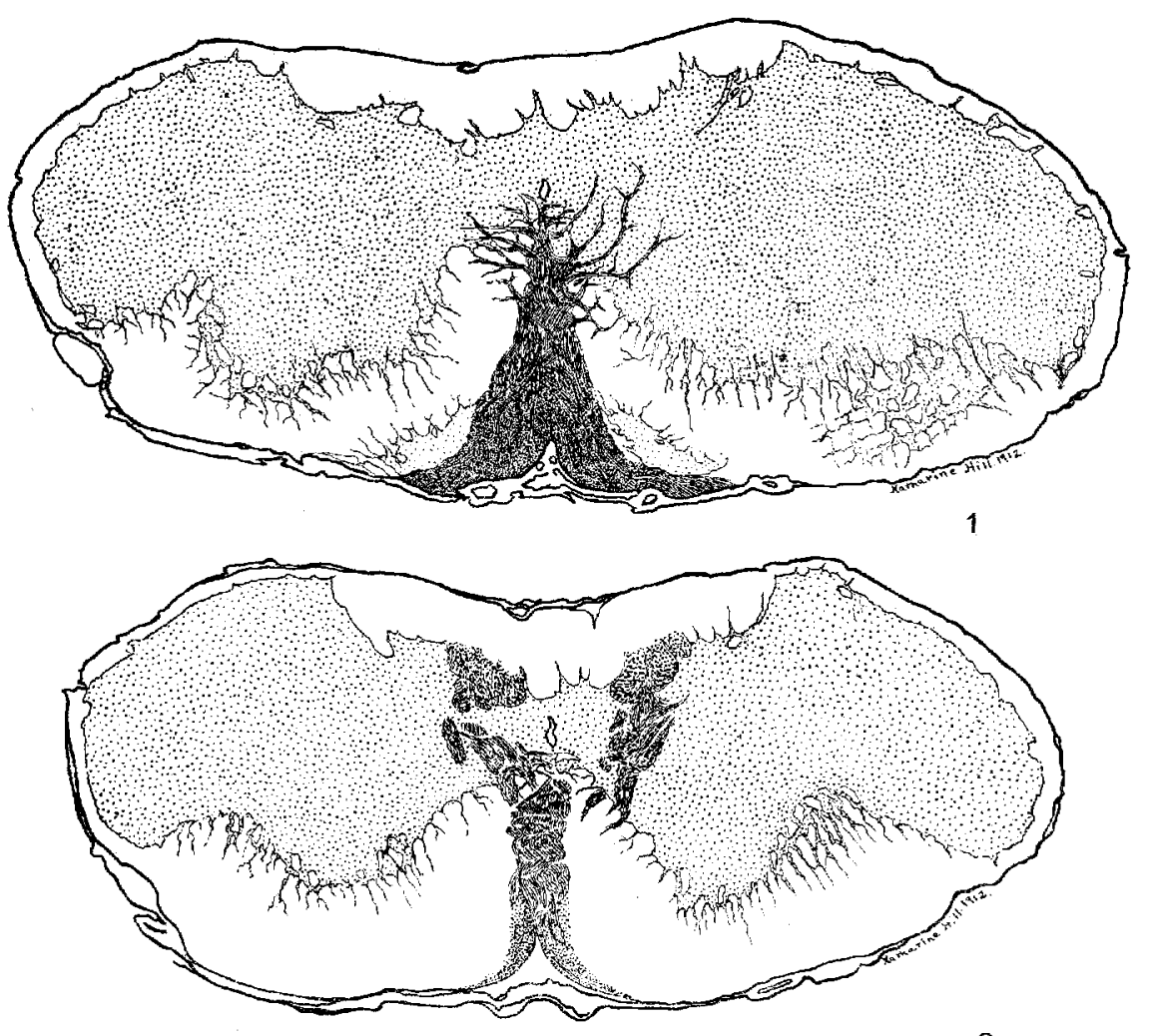

2

Fig. 1 Medulla oblongata, upper end of decussation of the pyramids. Ocu. 0 , Obj. 3 .

Fig. 2 Medulla oblongata, middle of the decussation of the pyramids. Ocu. 0, Obj. 3 .

decussation. The pyramids have disappeared from the ventral surface of the medulla; at a some of the lowest decussating fibers are indicated. The two large pyramidal fasciculi lie near the posterior median septum.

The pyramidal decussation differs from that in man, in that the fibers go over into the posterior instead of the lateral funiculus, 
and in that the decussation in the rat is complete. No pyramidal fibers run directly down on the same side into the anterior funiculus of the cord.

In the cervical region of the spinal cord (fig. $4, c .7$ ) these tracts occupy the ventral portion of the posterior funieuli and are closely
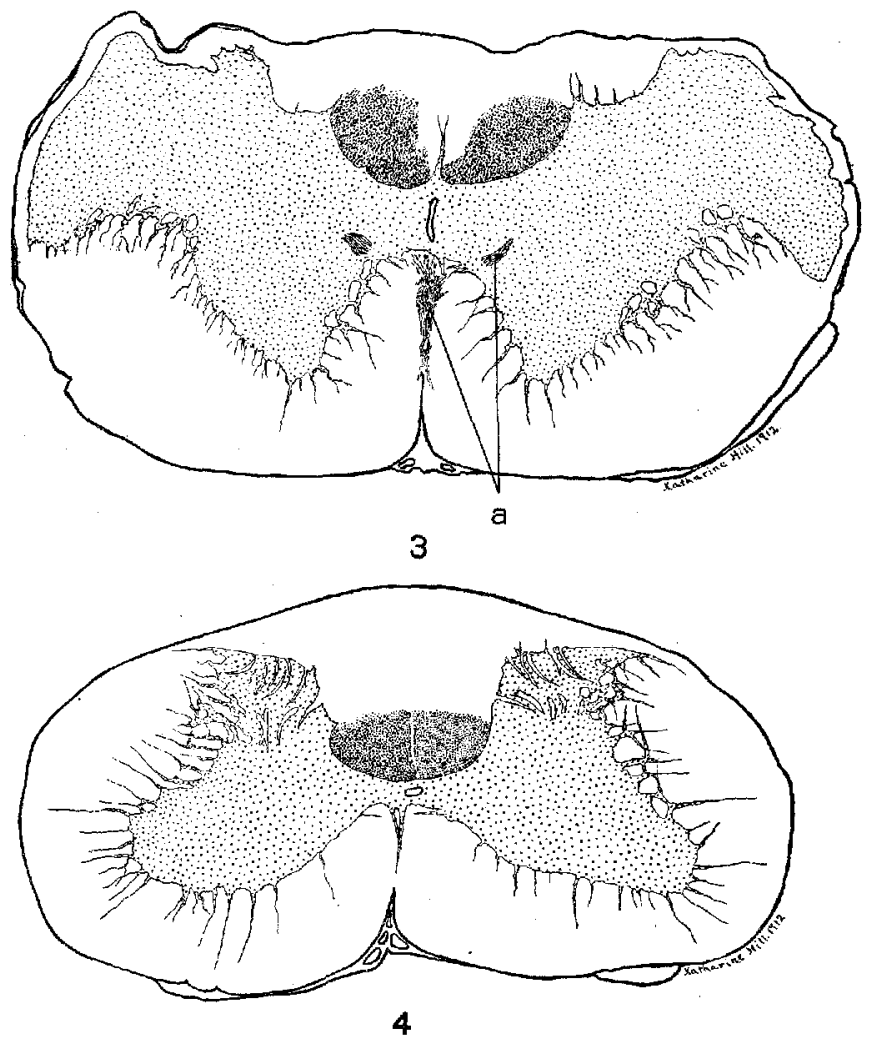

Fig. 3 Medulla oblongata, lower end of the decussation of the pyramids; $a$, lowest decussating fibers. Ocu. 0,0 bj. 3 .

Fig. 4 Seventh cervical segment of the spinal eord. Ocu. 0, Obj. 3.

approximated to each other and to the curved surface of the culumna posterior. Since the medial and posterior surfaces of of the bundle are almost at right angles to each other, the shape of the tract, as seen in sections through this level of the cord, is that of one-fourth of a circle. There is not as much intermingling 
of the pyramidal with surrounding fibers as one sees in the human cord. In the rat the tract stands out as sharply outlined in the preparations as it is in the drawings.

In the upper thoracic region the bundle changes its shape somewhat, since the posterior surface forms an acute angle with the medial surface; and the area occupied by the tract in sections of this part of the cord has the shape of an eighth part of a circle. Figure 5 was drawn from a Pal-Weigert preparation taken at

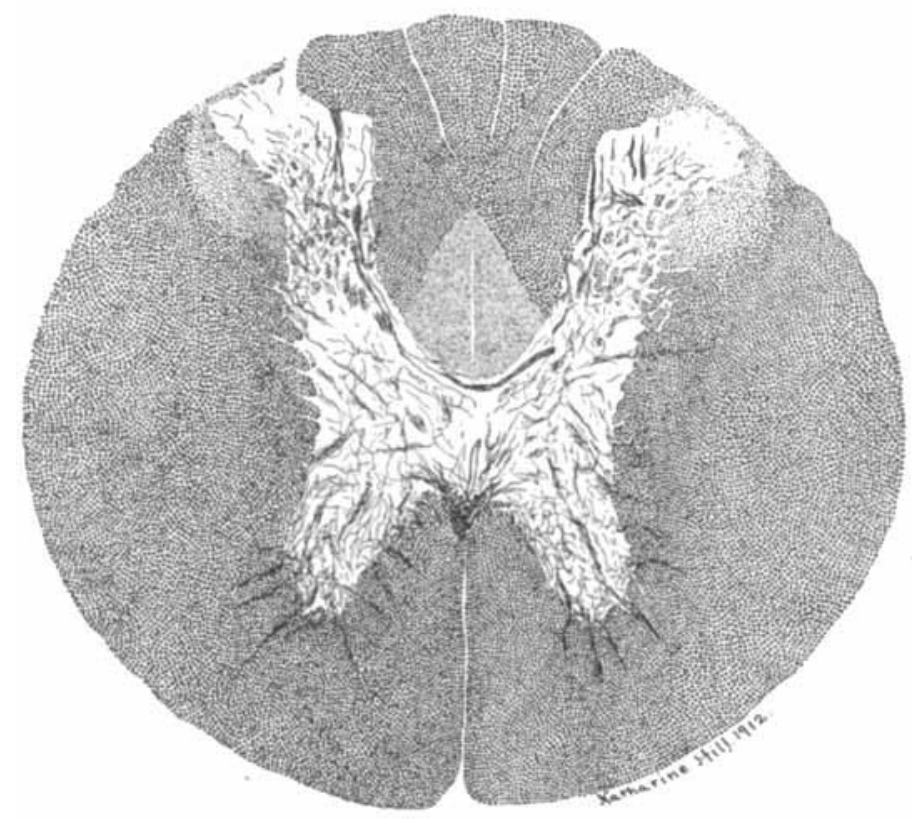

Fig. 5 Fourth thoracic segment of the spinal cord. Ocu. 3, Obj. 3.

about the level of the fourth thoracic segment and shows the tract clearly outlined by its fainter staining from the remainder of the white substance.

In the mid thoracic segments the bundle becomes rounded or oval in outline, and in the lower thoracic segments (T.12) it spreads out laterally along the posterior surface of the gray substance (fig. 6). In the upper lumbar region the outline of the tract is no longer so sharply indicated, and the tendency to spread 
out laterally is more pronounced. In the lower lumbar segments (fig. 7, L. 5) the fibers are diffusely scattered through the ventral part of the posterior funiculus and the tract has lost entirely its definite outline.

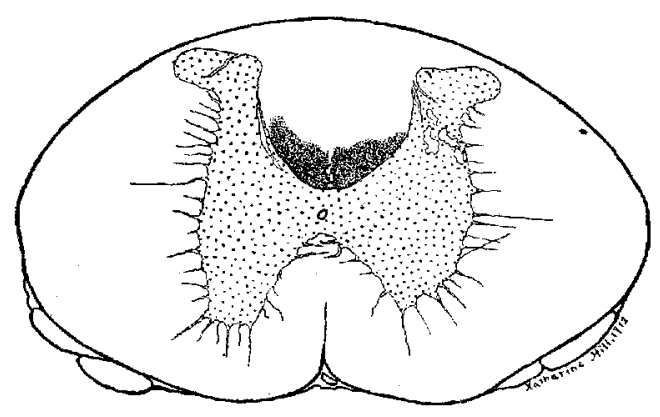

6

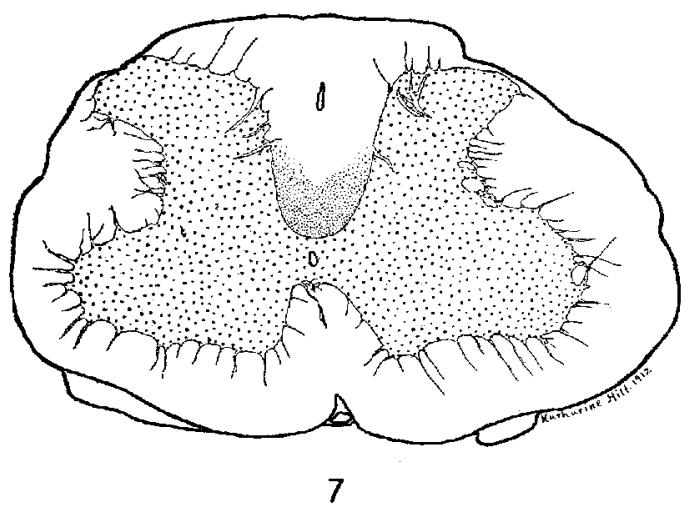

Fig. 6 Twelf th thoracie segment of the spinal cord. Ocu. 0, Obj. 3.

Fig. 7 Fifth lumbar segment of the spinal cord. Ocu, 0, Obj. 3 .

There is a progressive decrease in the size of the pyramidal tract as it runs caudad through the posterior funiculus of the spinal cord. This is seen on comparing the seventh cervical (fig. 4) with the twelfth thoracic segment (fig. 6). 


\section{STRUCTURE}

We turn now from the consideration of the tract as a whole to the characteristics of the individual fibers and shall learn why the tract stains so intensely with the pyridine-silver technique and so faintly with the Weigert methods. In pyridine-silver preparations of the spinal cord all the axons are stained; the larger ones are yellow, while the smaller ones are dark brown or black. The other elements of the white substance (such as myelin sheaths, neuroglia and blood vessels) are stained faintly or nor at all. Nearly all of the axons in the pyramidal fasciculus (fig. 8, a) are very small and these closely packed dark brown axons give the characteristic brown color to the fasciculus as a whole. This contrasts sharply with the structure seen in the remainder of the white substance of the cord (fig. $8, b$ ) where the large, light yellow axons, surrounded by thick unstained rings of myelin give rise to a lighter color and a more open structure. There are a few medium-sized axons in the pyramidal tract and a few of the very fne ones in the other fasciculi of the cord. Figures 8 and 9 were taken from the fourth thoracic segment of the spinal cord at the border of the pyramidal fasciculus.

As has been said, the pyrimidal tract takes a light grayish blue stain in Weigert preparations (fig. 5). It contains many very fine medullated fibers and a few of medium size (fig. 9,a). The myelin sheaths of the pyramidal axons are thinner than the sheaths on axons of the same size in other regions of the cord. Some are so thin and faintly stained that they are just recognizable. The medullated fibers do not occupy all the space in the tract but are separated from each other by unstained spaces. When we compare the Weigert and the pyridine-silver preparations of the same level of the cord we see that the axons in the pyramidal fasciculus (fig. $8, a$ ) are much more numerous than the myelin sheaths (fig. 9,a) and that the axons are more closely packed together. In any given section, therefore, many of the axons are without myelin sheaths. This is susceptible of two interpretations: either many of the pyramidal fibers are entirely non-medullated; or the myelin sheaths of the pyramidal fibers are 
interrupted, medullated and non-medullated stretches succeeding each other along the course of the same fiber. In any case, the medullation of the pyramidal tract in the white rat is incomplete, and such sheaths as are present are very thin. It is not possible to draw the same sharp line between entirely non-medullated and fully medullated fibers that can be drawn in the spinal nerves.

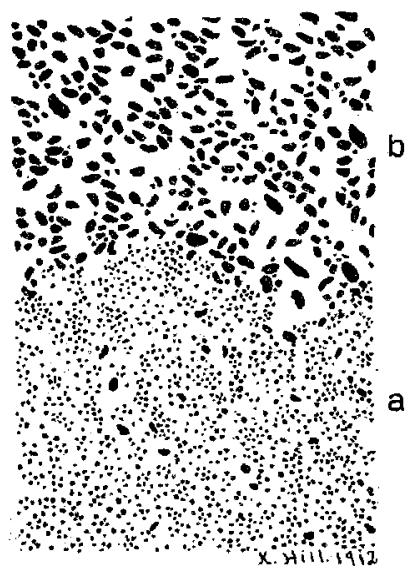

8

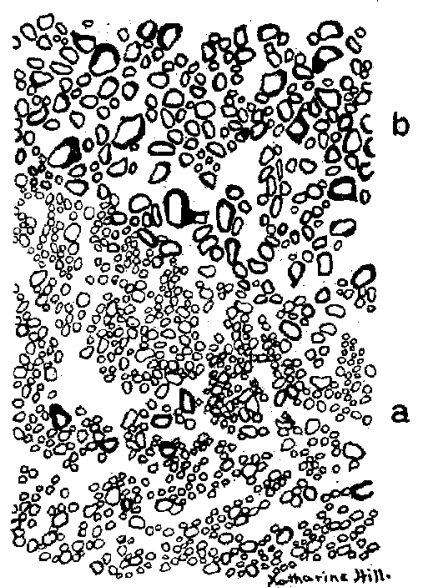

9

Fig. 8 An area from the fourth thoracic segment of the spinal cord at the boundary botween the pyramidal, $a$, and the cuneate, $b$, fasciculi; only axons are shown. Oou. 3 , Obj. $2 \mathrm{~mm}$.

Fig. 9 An area from the fourth thoracic segment of the spinal cord at the boundary between the pyramidal, $a$, and the cuneate, $b$, fasciculi; the myelin sheaths are shown. Oeu. $3 ;$ Obj. $2 \mathrm{~mm}$.

The same results are given by both the old Weigert and the Pal-Weigert methods. Care has been taken not to decolorize the smallest myelin sheaths. To avoid this, sections 8 to $10 \mu$ thick were used, and the decolorization was stopped just short of completion, leaving a diffuse light blue tint in the background. In these thin preparations it was possible to see dark blue myelin sheaths clcarly outlined against the lighter background. The number of sheaths seen in this way corresponded with the number seen in the more fully differentiated preparations. When a well differentiated preparation is stained with acid fuchsin a 
counter-stain of the axons is obtained. The pyramidal tract stains intensely with the fuchsin because of the predominance of axon substance in its composition.

Non-medullated fibers are also found in other parts of the white substance of the rat's spinal cord but are much less numerous than in the pyramidal fasciculus.

It should be added that all these observations were made on well developed adult rats, and are not to be explained by an immaturity of the individual animals employed.

These observations on the character of the fibers in the pyramidal tract of the white rat were made in connection with a search for the path within the spinal cord taken by the non-medullated fibers of the dorsal roots (Ranson '12). It is conceivable that they might run into the ventral portion of the posterior funiculus and ascend in the region occupied by the pyramidal tract; and since the number of non-medullated fibers from the dorsal roots is very considerable, such ascending fibers might represent all of the non-medullated fibers seen in this part of the cord. That is to say, the tract described in the first part of this paper might be a mixed one consisting of descending medullated fibers from the motor cortex and ascending non-medullated fibers from the dorsal roots.

In order to rule out this possibility, the following experiment was performed on adult albino rats. Under aseptic precautions the sciatic nerve was exposed in the upper part of the thigh, grasped with artery forceps and torn out of the pelvis. Five experiments were made. In one case two dorsal roots and their ganglia came away with the sciatic, in the remaining four only one root and ganglion. Each animal was killed after from twenty-four to twenty-eight days. No attempt was made at the autopsy to determine which of the roots associated with the sciatic was torn away in the operation. Pyridine-silver preparations were made of the lumboscaral portion of each of these cords. A varying degree of degeneration was seen in the last lumbar segments, depending upon the amount of damage done to the ganglia and roots when the sciatic was torn out. But in each case a very definite degenerated area could be seen, from which most of the 
axons had disappeared. Figure 10 illustrates the appearances seen in these sections. It will be observed that the posterior funiculus on the left side of the drawing is considerably smaller than that on the right and contains a degenerated area (a) reaching from the surface of the cord to the interval between the two pyramidal fasiculi. The area occupied by large undegenerated medullated fibers on the left side (b) is about one-half that on the right. But there is no clearly marked decrease in the size

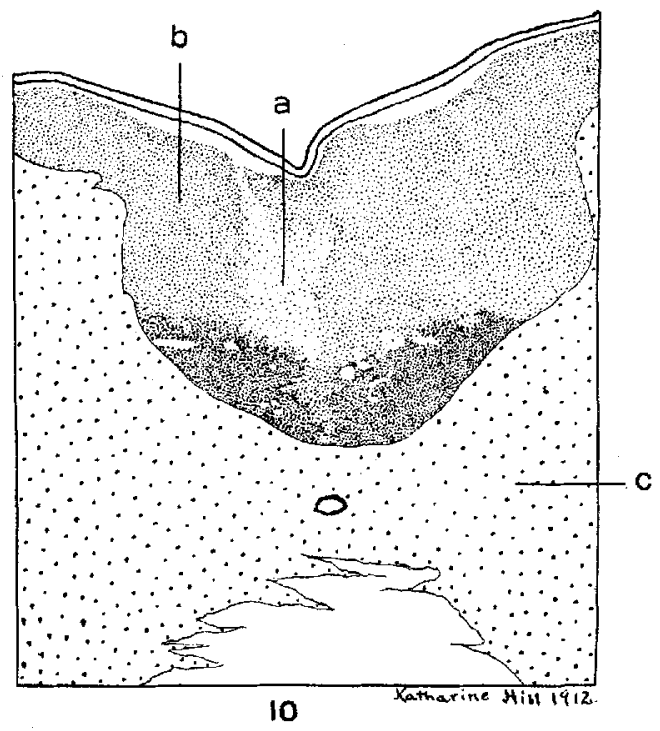

Fig 10 Part of a section through the lumbar portion of the spinal cord showing degeneration, $a$, in the cuneate fasciculus. Undegenerated portion of the cuneate fasciculus, $b$; gray substance $c$. Ocu. 3 , Obj. 3 .

of the pyramidal fasciculus nor any noticeable degeneration of the axons within its area.

In order to compare more accurately the areas occupied by the pyramidal fasciculi on the normal and operated sides, these areas were roughly computed in sixteen successive sections. This was done by tracing the outlines of the tracts with the camera lucida on millimeter paper, and determining the number of square millimeters covered by the areas thus projected. It was found that the tract on the operated side was 3 per cent smaller than that 
on the normal side, a decrease which could be accounted for by the presence of a few dorsal root fibers within the area of the tract. Since the tract decreased so little in size and since there were no other evidences of degeneration within its territory, it is obvious that the non-medullatd fibers which it contains do not belong to another system arising in the dorsal root ganglia. These experiments also serve to emphasize the sharpness with which the regions occupied by the pyramidal tracts are limited in the white rat.

Watson ('03) noticed that in the Pal-Weigert preparations of the spinal cord of the adult albino rat the pyramidal fasciculus was only slightly stained and he attributed this to a supposedly different chemical composition of the myelin in the sheaths of these fibers. Miss King ('10) states that, when compared with the Marchi preparations of the pyramidal fasciculus in the rabbit, cat and $\operatorname{dog}$, the Marchi preparations of this tract in the rat reveal a striking paucity of fibers "so that in this animal the socalled primary motor path is probably only of secondary importance." In view of the incomplete medullation of the pyramidal tract in the rat it is easy to understand Miss King's results. Although there is an abundance of axons there are few well medullated fibers, such as would respond readily to the Marchi stain. Just how far the incomplete medullation of this tract is an indication of an incomplete development of its function is a matter which it would be very difficult to decide. Attention has been called to the peculiar light staining of this fasciculus in Weigcrt preparations of the spinal cord of animals belonging to widely separated groups. Ziehen ('99 and '00) mentions it as occurring in the pseudochirus, the sheep and the rat. Dräseke ('04) observed that in the mole the pyramidal fibers lose their myelin sheaths as they pass from the medulla into the anterior funiculus of the spinal cord, into which they go without decussation. Here they form a medially placed oval field somewhat ventral to the anterior commissure. This oval area is very faintly stained in Weigert preparations and is almost devoid of medullated fibers. Bischoff ('00) states that in the hedgehog the pyramidal fibers are so fine and possess so delicate a myelin sheath that the Marchi stain 
does not give good results. He could follow only a few degenerating fibers into the spinal cord where they lay in the homolateral anterior funiculus. They could not be traced beyond the upper cervical segments. These findings seem to indicate a condition in the hedgehog similar to that found by Dräseke in the mole.

It would seem, therefore, that the incomplete medullation of the pyramidal tract in the rat is not a characteristic peculiar to these animals but is related to similar conditions in at least some marsupials (pseudochirus), some species of insectivora (mole, hedgehog), some other rodents (guinea-pig) and some ungulates (sheep). Since, however, the observations on these other animals were confined to myelin sheath stains it seems desirable to make a comparative study of the question with an axon stain. Such an investigation is now under way.

In man, medullation of the pyramidal tracts begins shortly before birth and is not completed until the second year. It is obvious that this tardy medullation is rendered more significant in the light of these facts concerning the condition of this tract in some of the lower animals.

\section{BIBLIOGRAPHY}

Bechrerew, W., 1890 Ueber die verschiedenen Lagen und Dimensionen der Pyramidenbahnen beim Menschen und den Thieren. Neurol. Centralbl., Bd. 9, \$. 738.

Bischoff, E. 1900 Beitrag zur Anatomie des Igelgehirnes. Anat. Anz., Bd. 18, p. 348 .

Dräskef, J. 1904 Zur Kenntnis des Rückenmarks und der Pyramidenbahnen von Talpa europaea. Monatsthr. f. Psy. u. Neurol., Bd. 15, p. 401.

Goldstein, G. 1904 Zur vergleichenden Anatomie der Pyramidenbahn. Anat. Anz., Bd. 24, p. 451.

King, Jessie L. 1910 The cortico-spinal tract of the rat. Anat. Ree., vol. 4, p. 245 .

von Lexнossék, M. 1889 Über die Pyramidenbahnen im Rüchenmarke einiger Säugetiere. Anat. Anz., Bd. 4, S. 208.

Ransox, S. W. 1912 The structure of the spinal ganglia and of the spinal nerves. Tour Comp. Neur., vol, 20, p. 159. 
Sfitzka, F. C. 1886 The comparative anatomy of the pyramid tract. Iour. Comp. Medicine, vol. 7, p. 1.

Stieda, L. 1869 Studien über das centrale Nervensystem der Vögel und Säugethiere. Zcitschr. f. wissensch. Zoologie, Bd. 19, S. 1.

VAN der VloRT, 1906 Ueber den Verlauf der Pyramidenbahn bei niederen Säugetieren. Anat. Anz., Bd. 29, p. 113.

Watson, J. B. 1903 Animal education. Chicago, 1903.

ZIEHen, Th. 1899 Zur vergleichenden Anatomie der Pyramidenbahn. Anat. Anz., Bd. 16, p. 446.

1900 Ueber die Pyramidenkreuzung des Schafes. Anat. Anz., Bd. 17 , p. 237. 